\title{
Modeling with ontologies design patterns: credit risk scorecard as a case study
}

\author{
Jalil El Hassouni ${ }^{1}$, Abderrahim El Qadi ${ }^{2}$, Mehdi Bazzi ${ }^{3}$, Mohamed El Haziti ${ }^{4}$ \\ ${ }^{1,4}$ LRIT-CNRST (URAC'29), Faculty of Sciences Rabat IT Center, Mohammed V University, Morocco \\ ${ }^{2}$ High School of Technology in Sale, Mohammed V University, Morocco \\ ${ }^{3}$ LIAD, Faculty of Sciences of Ain Choc, Hassan II University, Morocco
}

\begin{tabular}{l} 
Article Info \\
\hline Article history: \\
Received Feb 20, 2019 \\
Revised May 31, 2019 \\
Accepted Jul 30, 2019 \\
\hline Keywords: \\
Credit risk Scorecard \\
Decision support system \\
Ontology design patterns
\end{tabular}

Article Info

Received Feb 20, 2019

Revised May 31, 2019

Keywords:

Decision support system

Ontology design patterns

\begin{abstract}
This paper proposes an ontological scorecard model for credit risk management. The purpose of the credit scoring model is to reduce the possibility of potential losses with regard to issued loans. Loans are provided according to strict criteria which contain information about the client, loan structure, the purpose, repayment source and collateral. Several techniques have been used for credit risk assessment before granting a loan. Ontology design patterns are used here to enable the implementation of domain knowledge using the OWL rules and to improve the decision-making process in credit monitoring. The modeling of our ontology will make the data publication simpler and graph structures intuitive, thus making its reusability and expandability easier.
\end{abstract}

Copyright $@ 2020$ Institute of Advanced Engineering and Science. All rights reserved.

Corresponding Author:

Jalil El Hassouni, LRIT-CNRST (URAC'29),

Faculty of Sciences Rabat IT Center,

Mohammed V University, Rabat, Morocco.

Email: jalil.elhassouni@gmail.com

\section{INTRODUCTION}

In the last decade the amount of data generated on a daily basis has exponentially exploded. Numerous efforts have been undertaken to provide the industry and research fields with expanding technologies for facilitating the collection, treatment and sharing of massive data. This poses unprecedented challenges for the use of these technologies, especialy in the banking sector. Therefore, the central bank governors of the G10 and Switzerland created the Basel Committee on Banking Supervision (BCBS) in order to assist them in monitoring and exchanging data tasks based on common approaches and standards. BCBS reports that one of the reasons for the financial crisis of 2008 was associated with problems in data architecture and information technology infrastructure. Moreover, inadequate or non-existent of common vocabulary has created a gap in common semantics. As a consequence, for many banking systems, assertions or decisions that depend on the data cannot be reliable.

In the talk at 2010, Gov 2.0 Expo in Washington, D.C., Tim Berners-Lee suggested awarding stars for any kind of sharing data [1]. Therefore, governments earn three stars for sharing data on the web a non proprietary format, for stars on putting it in linked data and a full five stars for connection data to other data. Following the recommendations of BCBS and Tim Berners-Lee, an ontology-based approach has been proposed to assist different financial institute in standardizing, monitoring, and exchanging data in credit risk field.

Ontologies have a lifecycle: they are created, implemented, evaluated, fixed, exploited, and reused like any artifact. Numerous methodologies and methods for developing and modeling ontologies have been proposed in recent years; yet, none of them constitutes a normalized reference [2]. In this paper, we use 
ontology design patterns (ODPs) for modeling credit risk scorecard as worked example. ODPs are reusable solutions for recurrent ontology modeling problems, which permits our ontology to be reused and expanded. In $[3,4]$ first proposed this idea with the aim to clearly design ontologies used as the basis for building other ontologies and to foresee the effect of changes or extensions to them. It is also rarely the case that you, as an ontology engineer, having the set of requirements for your ontology engineering task at hand, will fully agree with all the ontological commitments that are made in such a large ontology. However, not reusing any wellestablished practices at all, and not aligning your ontology partly with existing ontologies will create problems in the interoperability, and potentially also, understandability of your ontology. Hence, there is a trade-off between interoperability, on the one hand, and over-commitment and conflicting requirements on the other hand. This is where ODPs as small "building blocks" offer one way to manage this trade-off. ODPs carry the promise of better integration and interoperability of data across various domains [5]. There are several types of ODPs and they can be reused and applied in many different ways. [6] has identified them and grouped them into six families shown in Figure 1: Structured OPs, Correspondence OPs, Content OPs (CPs), Reasoning OPs, Presentation OPs, and Lexico-Syntactic OPs.

A Content OPs (CP) can be considered roughly as analogous to a software design pattern with the added benefit that it includes a reference base implementation (in the form of an OWL building block) ready for immediate customization [7].

[6] noted that each CPs is associated with a catalogue entry including the following set of information fields. Name provides a name for the pattern; Intent describes the generic use case addressed by pattern; Competency Questions (CQs) contains examples of CQ that the knowledge base associated with the CPs needs to address; Diagram depicts a UML class diagram representing the pattern; Elements describes the classes and relations included in the pattern. Also, CPs is described with the Scenario, Consequence, Known, Extracted from/Reengineered from and Related patterns. Thereby, it should be noted that, to our knowledge, there are two ways to model with ODPs - The first approach is called the eXtreme Design Methodology [8] and the second, which we follow here, is mostly inspired from [9] and [10]. The later consists of:

1) Formulating use case(s) for which the ODP is intended.

2) Modeling diagram and the appropriate logical axiomatization identified from the use case descriptions.

3) Verifying the resulting ODP to ensure its quality by checking the set of its axioms, computing some of its logical consequences, and populating it with sample data, which sometimes exposes shortcomings.

In this work, we have chosen to use CPs as defined in the NeoN Project to model our ontologies, as this is the most common type of ODPs with some 100+ patterns published [11].

The remainder of this paper is organized as follows: in section 2, we introduce the related works. In section 3, we discuss the credit risk scorecard concepts, then, we show the process of creating an ontology credit risk scorecard pattern for credit monitoring in section 4. In section 5, we evaluate our ontology model, and in the last section we conclude with discussing future work.

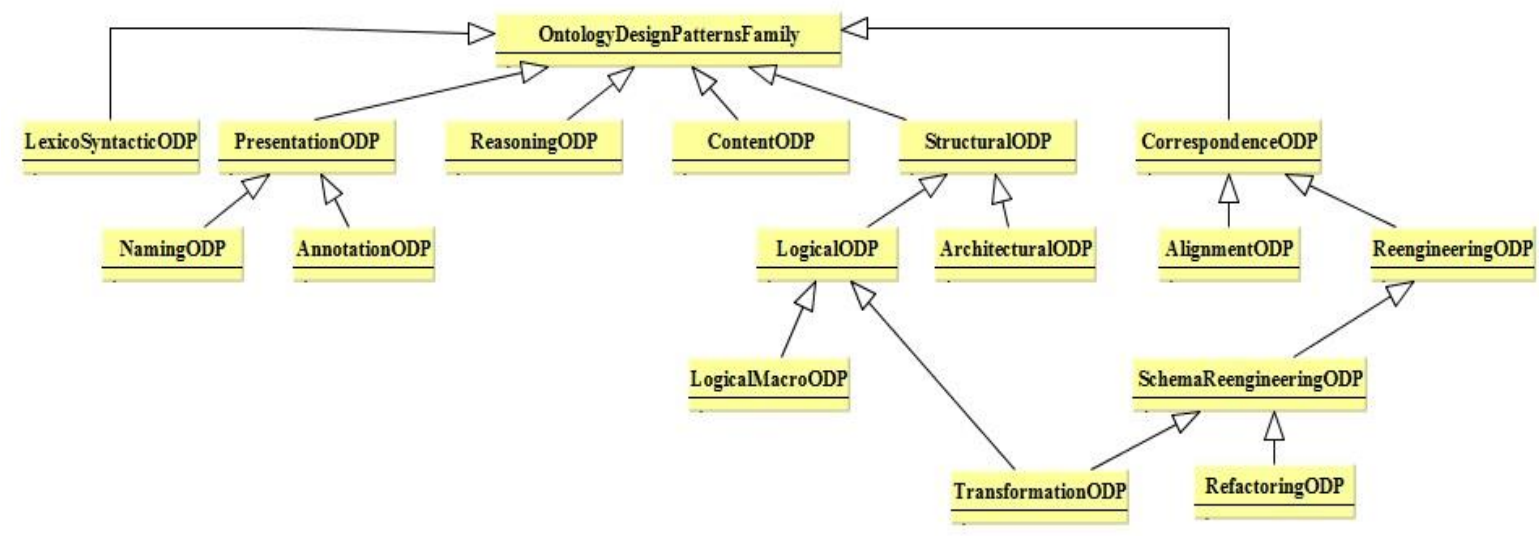

Figure 1. Ontology design pattern's family

\section{RELATED WORK}

In recent years, ontologies have been increasingly used in industry and research fieldsfor different purposes. The development ofsemmantic domain ontology can help in reducing common problems and ambiguities linked to tag based systems [12]. Ontologies representing the domain knowledge have been used 
to guide the design of the application and to supply the system with semantic technologies possibilities [13-16]. The general ontology that models the credit risk management process and two specific ontologies have been proposed [17]. One of these specific models the process of credit allocation to clients, while the second displays necessary concepts for monitoring of a credit system. Ontologies are beneficial when they are used in decision support systems [18]. The later work proposes an integrated ontological model for evaluating client applications which incorporates both: the default risk of investment and the development component of the investment. [19] proposes an approach for the conceptualization and the definition of business rules present in governance policies of the Brazilian financial system, more specifically those related to risk management. It proposes an ontology called Onto-Bacen that expresses the concepts (and their relationships) of this domain, and by using inference algorithms, which can verify the compliance of hypothetical financial institutions with those policies.

Decision making is one of the main research themes of systems' science, and decision support systems (DSS) were developed in many areas; e.g., management decision making, group decision making, etc. Authors in [20] developed an ontology based on decision support systems to assess the risk factors and provide appropriate treatment suggestions for diabetic patients. [21] presents an ontology-driven method for multi-criteria decision making that explicitly focuses on ensuring that the consequences of each choice are considered. In [22] the authors combine between mobile technology and fuzzy ontology and group decisionmaking algorithms, to facilitate the mobilization of knowledge, giving the user the possibility to get decision making support from the dynamic and massive data through their mobile services.

[23] Attempts to present an ontological risk analysis in which the authors integrate three different perspectives on risk: (I) risk as a quantitative notion, (II) risk as a chain of events that impacts an agent's goals, (III) risk as the relationship of ascribing risk. Despite their effort, their proposal remains a superficial and simplified approach. Moreover, the implementation of this ontology in the analysis of risk in a specific domain, such as credit risk, remains a tedious task that may not succeed even though the life cycle of the development of the ontology is not specified and the ontology is not validated.

However, the above-mentioned solutions are not conclusive. Among their major draw backs we can cite: requiring a lot of manpower and time to manually integrate them, designed and modeled for specific contexts with strong commitments and great details rendering their reusability and expandability difficult. To address some of these limitations we developed a flexible conceptual architecture based on ontology design patterns.

\section{CREDIT RISK SCORECARD}

\subsection{Requirements and Use Case}

The credit-granting process leads to two choices: granting a loan to a new customer or declining his application. The purpose of the credit risk scorecard is defined as "the assignment quantitative measure to a potential borrower to provide an estimation of its capacity to repay a loan" [24]. Usually, logistic regression is the most used techniques to build credit risk scorecard. More recently, artificial intelligence techniques like expert systems and neural networks have been used [25]. All of them involve establishing and quantifying the relationship between the characteristics and good/bad performance (target).

The use case that drives our modeling allows the credit analyst to calculate the number of points of each applicant Table 1(a). It also provides them with a decision support tool Table 1(b) which allows them to immediately reach an opinion on the credit allocation.

The modeling has to be as general as possible so that it becomes possible to add information from any sources (web, credit bureau data, financial ratios, social networks...etc.). The schema has to be robust, extendable, and easy to be maintained and updated. In order to achieve these objectives the credit risk scorecard and decision support tool are modeled with ontology design patterns.

The ontology is built to address a set of requirements; in fact, it is evaluated against its corresponding requirements specification. These requirements can be defined through appropriate CQs, which define the scope of ontology. Some typical CQs are listed below:

1) Which variables take part in the credit risk scorecard?

2) Which credit risk scorecard does this variable participate in?

3) What is the value of a given category?

4) What is the score band of low, medium and high scores?

5) What does good and bad client mean?

6) How many counts of a client type are bad?

7) How many counts of a client type are good?

8) What is the risk of an applicant who is under 25 applying for credit for the first time at the institution with no other credit, no non-payments, with an account having slightly positive balance (but less than 
$€ 200$ ), with a small amount of savings (less than €500), and without a guarantor applying for credit for 36 months?

9) What is the risk of an applicant aged over 25 with credits in competing institutions, without nonpayments, with an account having an average balance of more than $€ 200$, with more than $€ 500$ in savings, and without a guarantor applying credit for 12 months?

Table 1(a). Example of credit scoring scorecard

\begin{tabular}{ccc}
\hline Variable & ClassVal0 & Nbpoints \\
\hline Age & $>25$ years & 0 \\
& $<=25$ years & 8 \\
Other_credits & No credit & 0 \\
& Other banks or & 7 \\
& institution & \\
Accounts & No cheking account & 0 \\
& CA $>=200$ euros & 13 \\
& CA [0-200 euros] & 19 \\
& CA < 0 euros & 25 \\
Credit_duration & $<=15$ months & 0 \\
& $16-36$ months & 13 \\
& $>36$ months & 18 \\
Savings & No savings or $>500$ & 0 \\
& euros & 8 \\
Guarantees & $<500$ euros & \\
& Guarantor & 0 \\
Credit_history & No Guarantor & 21 \\
& No credit at any time & 0 \\
& Credits without delay & 6 \\
& Creditswithnon- & 13 \\
& payments &
\end{tabular}

Table 1(b). Example of credit scoring decision support

\begin{tabular}{cccc}
\multicolumn{4}{c}{ tool } \\
\hline Nbpoints & \multicolumn{3}{c}{ Credit } \\
\hline Frequency & Good & Bad & Total \\
Percent & & & \\
Row Pct & & & \\
Low risk & 389 & 37 & 426 \\
& 38.90 & 3.70 & 42.60 \\
& 91.31 & 8.69 & \\
Medium risk & 239 & 137 & 376 \\
& 23.90 & 13.70 & 37.60 \\
& 63.56 & 36.44 & \\
High risk & 72 & 126 & 198 \\
& 7.20 & 12.60 & 19.80 \\
& 36.36 & 63.64 & \\
Total & 700 & 300 & 1000 \\
& 70.00 & 30.00 & 100.00 \\
\hline
\end{tabular}

\subsection{Data Sources}

Once we have a set of CQs as listed previously, we take a closer look at the data sources and the data structure. The used data set is credit risk scorecard Table 1(a) and credit scoring decision support tool Table 1(b) [26] which are the result of credit scoring modeling by logistic regression applied to 'German credit data'. The latter contains 1000 consumer credit files of which 700 'Good' applicants (no nonpayments) and its 300 'Bad' applicants (non-payments) and 19 independent variables.

The credit risk scorecard contains the selected variables, their division into categories (ClassVal0) and the weight per category for each variable (nbpoints). The decision support tool is divided in three score bands (low risk, medium risk, high risk); each band contains the number and the percentage of good and bad applicants.

The credit risk scorecard will make it possible to respond to some CQs, e.g., questions (1) and (2). However, the credit risk scorecard alone will not allow the possibility of addressing CQs as in numbers (5), (6), (7) and (8) requiring the decision support tool, and (4) which would require more fine-grained information about the objectives of the project for which the credit risk scorecard is developed.

For additional information, possible sources are plentiful, mainly when the applicant's file needs close analysis and examination, e.g., credit bureau data, social network, demographic data. For the moment, attention will be given to model existing sources, credit risk scorecard and decision support tool. However, at the same time our modeling should be expandable as possible to include other data sources.

\section{CREDIT SCORECARD MODELING}

\subsection{Ontology Modeling}

We have a working group with a mix of participants: domain expert, participants specializing in data base addressed by modeling, and ontology engineer working on ODP-based modeling process. Reconciling the differing perceptions of domain expert to their topic, credit risk scorecard and decision support tool, - as data providers - and the CQs cited above leads to an expandable scope. Thus, requires data integration which is part of our use case. The modeling of our ontology will make the data publication simpler and graph structures intuitive, as will as making its reusability easier [10].

Based on the credit riskscorecard and decision support tool formats discussed earlier, and based on our CQs, we can identify several key notions which will be in need of modeling :agent, event, variables, categories, decision support tool, credit risk scorecard support tool and credit risk scorecard. 
Let us start with the notion of the Agent. In order to borrow from best practices and realize that "being an agent" is actually a role which an agent can take (e.g. Developer, Analyst, and Manager Etc.), for a certain period of time. We therefore reuse the common ontology design pattern for this purpose, which is depicted in Figure 2 in exactly the form in which it is used, e.g., in [27]. We opted for adapting this pattern for our specific case, leaving most of the things untouched, while avoiding overgeneralizations. The resulting pattern is depicted in Figure 3. Note that we have introduced three different agent roles: Analyst, Developer and Manager. The yellow frames indicate that a more complex entity (a pattern in its own right) could stand in place of the frame. The latter could be modeled with a more fine-grained model, or alternatively, an existing ODP could be directly used instead.

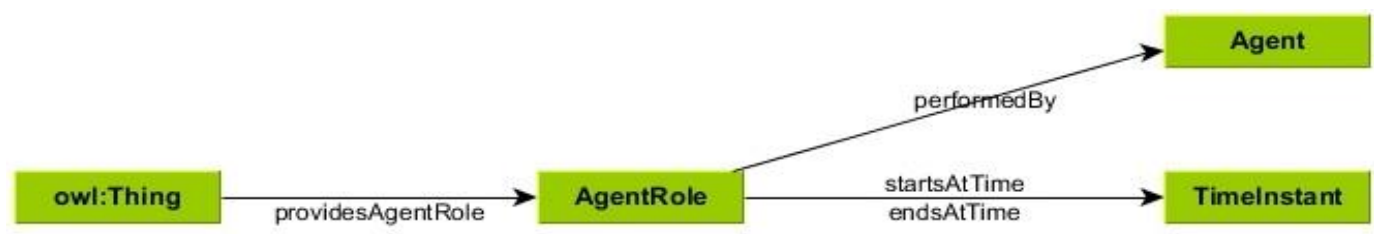

Figure 2. The agent role Content ODP's UML graphical representation

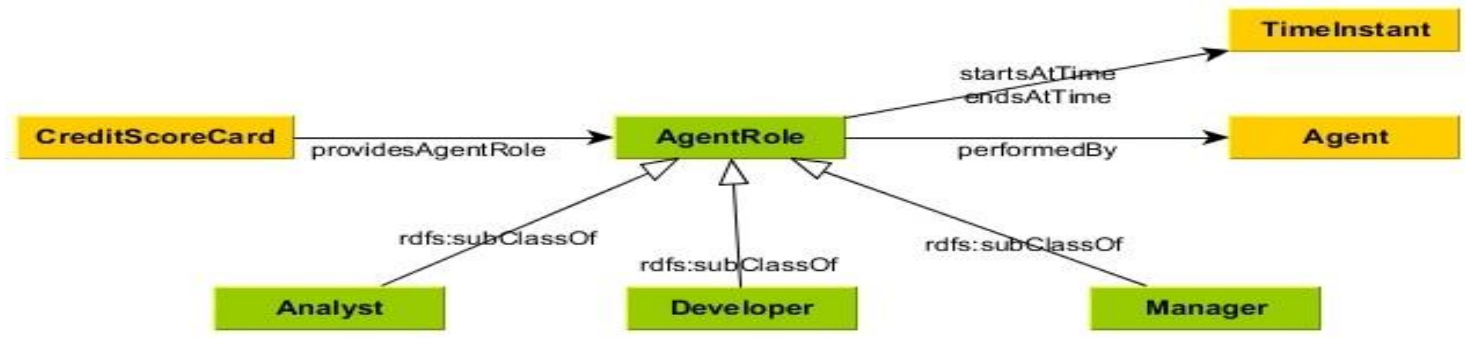

Figure 3. Adapted agent role ODP for credit risk scorecard player roles UML graphical representation

Let us turn our attention to credit risk scorecard, from the viewpoint of the team's domain expert, the edition and validation of credit risk scorecard is an event, or alternatively as a step of credit risk scorecard development Figure 4. Therefore we suggest to reuse a generic event pattern, such as the one depicted in Figure 5, in exactly the form in which it is used, e.g. in [27].

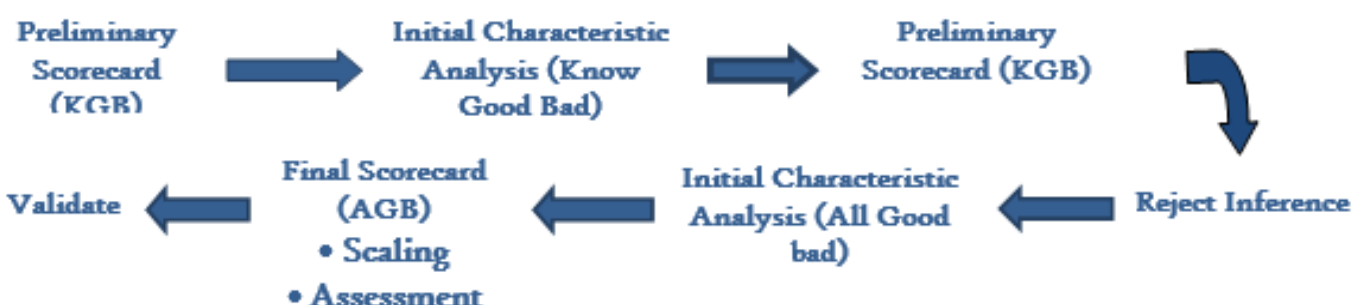

Figure 4. Credit risk scorecard development steps

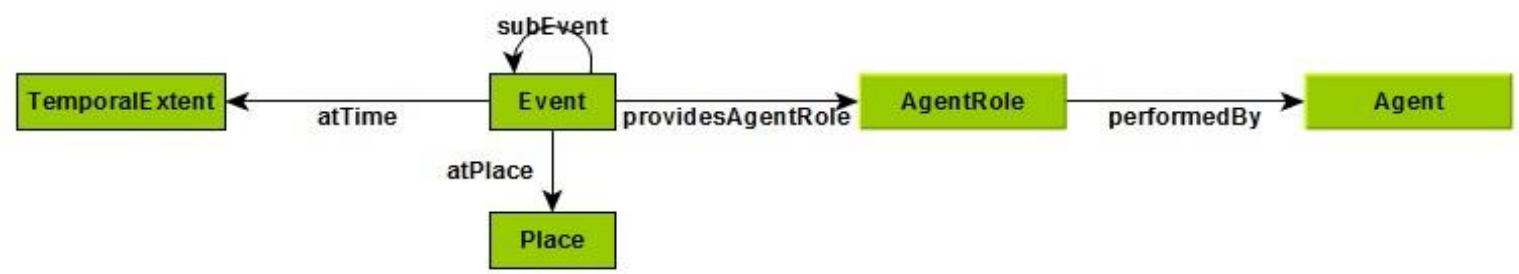

Figure 5. The event Content ODP's UML graphical representation 
As stated earlier, we specialize the pattern for our specific purpose, the result of which is depicted in Figure 6. Note that we linked directly the place to string containing the name of the place in which the credit risk scorecard was developed. The development of credit risk scorecard can be in-house or by external vendors. In contrast, if we had directly linked credit risk scorecard to the string containing the name of modelization algorithm, we would not have to provide an extension in the future. At the same time, we reuse the agent role pattern. However, the sub-event Figure 4 and temporal information are not treated at this stage, yet we intend to provide the possibility to make this extension later without having to change anything already modeled.

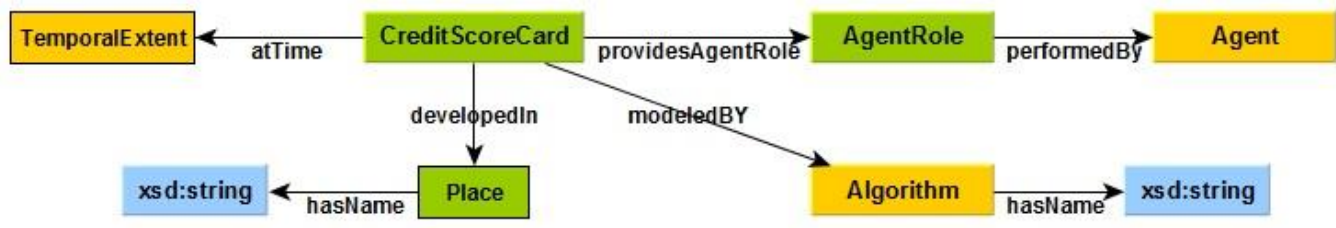

Figure 6. The credit risk scorecard as event

The next part we deal with is the variables; since we have settled for viewing the credit risk scorecard as event, the variables are naturally participants in the event. Therefore, we suggest reusing a generic participation pattern such as the one depicted in Figure 7(a) in exactly the form in which it is used, e.g., in. Following the same approach, we adapt this pattern to fit our specific case, so we prefer to leave most of the things untouched. The resulting pattern is depicted in Figure 7(b).

Considered from the viewpoint of our team's domain expert, the categories are classifications of variables. Therefore, we suggest reusing a generic classification pattern such as the one depicted in Figure 8(a) in exactly the form in which it is used, e.g., in. We intend to adapt this pattern for our specific case. The resulting pattern is depicted in Figure 8(b).

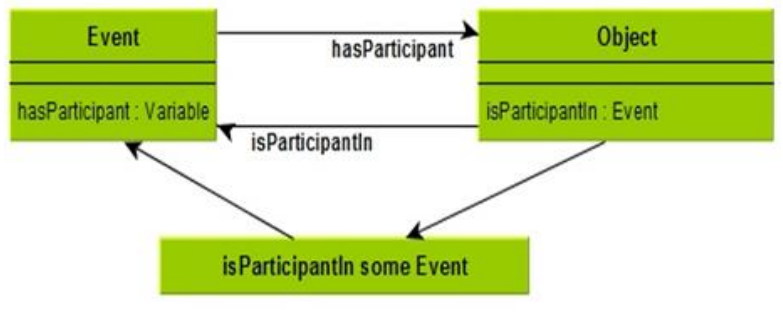

Figure 7(a). The participation Content ODP's UML graphical representation

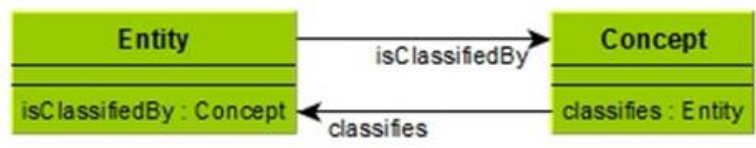

Figure 8(a). The classification content ODP'sUML graphic representation

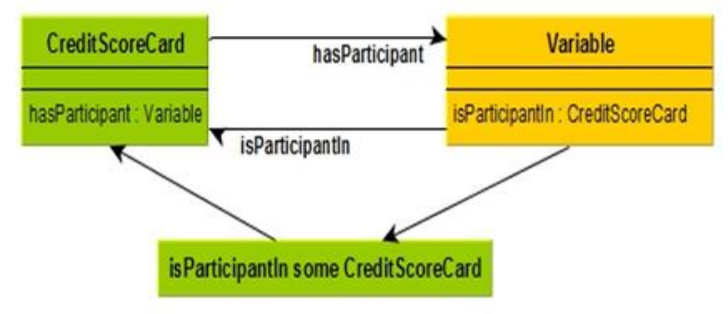

Figure 7(b). The variables as participation

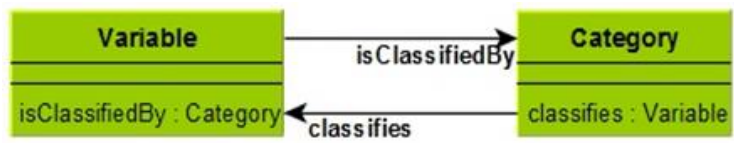

Figure $8(\mathrm{~b})$. The categories as classification

Moving to credit scoring decision support tool, the numbers of points are divided into bands. Therefore, the band risks (low, medium, high) are classifications of credit scoring decision support tool. Here, as in the previous case, we suggest to reuse a generic classification pattern depicted in Figure 8(a). The resulting pattern is depicted in Figure 9. It is worthy of note that we have introduced three different Band risks, two different Clients (Good and Bad) and the credit risk scorecard objectives. As mentioned above, and regarding future extension, we do not directly link client and credit risk scorecard objectives to strings containing the definition of client and credit risk scorecard objectives. 


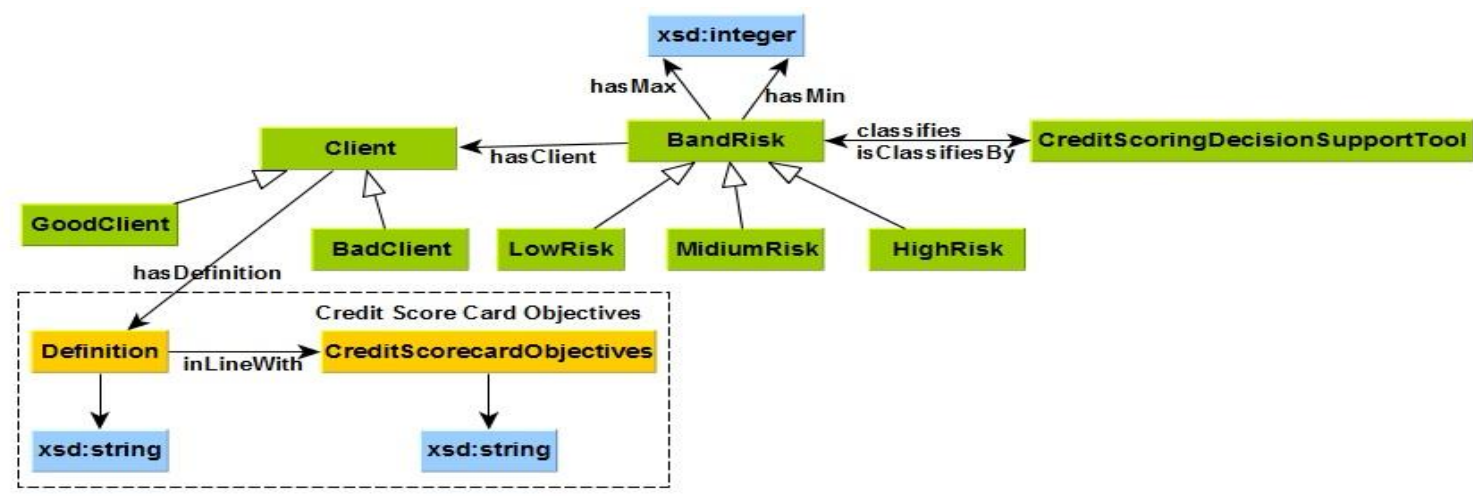

Figure 9. The credit risk scoring decision support tool as a pattern

\subsection{Relating Things Together}

Finally, after the development of each piece of the whole credit risk scorecard, we intend to assemble all the pieces, producing the result depicted in Figure 10. Note that we directly linked credit risk scorecard to credit risk scorecard objectives and credit scoring decision support tool.

\subsection{Validation of The Ontology}

Apart from the evaluation undertaken by the domain experts, our ontology must be validated in order to develop a reference and reusable ontology in the credit risk field. Therefore, since another credit risk ontology was not available in order to make a feasible comparison, we decided to use an approach in the ontology evaluation field. According to [28], any approach of ontology evaluation has to take into consideration several criteria: accuracy, completeness, conciseness, adaptability, clarity, computational efficiency and consistency.

Many approaches in the ontology evaluation field have been discussed in [29]. Therefore, we opted for an automatic online evaluation framework for OWL ontologies which contains a common pitfalls catalogue [29] and which respects most of the criteria of ontology evaluation [28]. The online framework evaluation detected no errors in any of the indicators supported by OOPS. Since we used the Protégé ontology editor, we also opted for HermiT1.3.8.413 [30] reasoners bundled in it for testing its consistency. The result revealed that no contradictions have been found in our ontology.

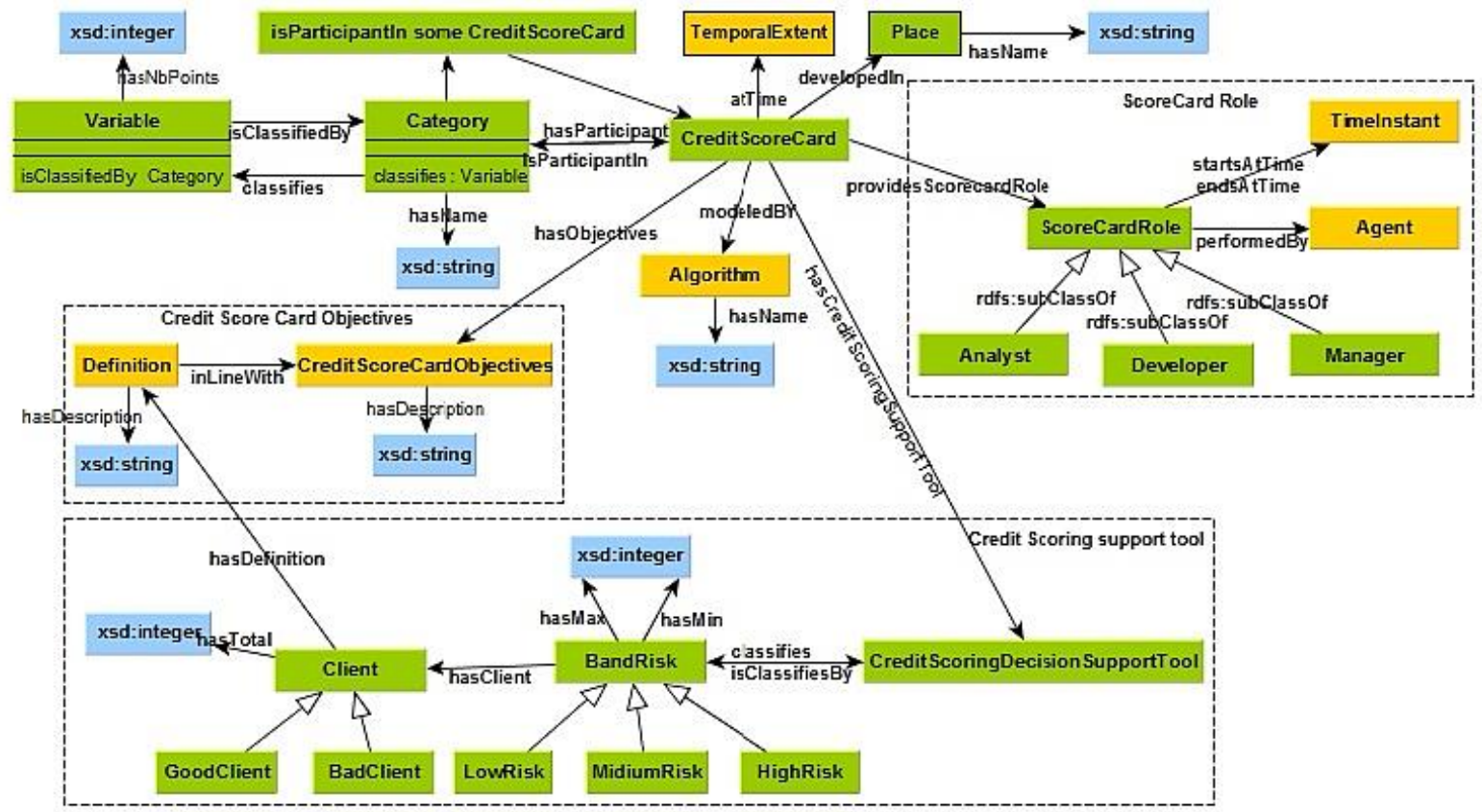

Figure 10. The credit risk scorecard model 


\section{CREDIT RISK ASSESSMENT}

The system consists of two main ontologies, the credit risk scorecard ontology and the applicant profile ontology. They are implemented using Web Ontology Language (OWL), with the Protégé tool [31].

The proposed reasoning algorithm for the applicant analysis is illustrated in Figure 11.

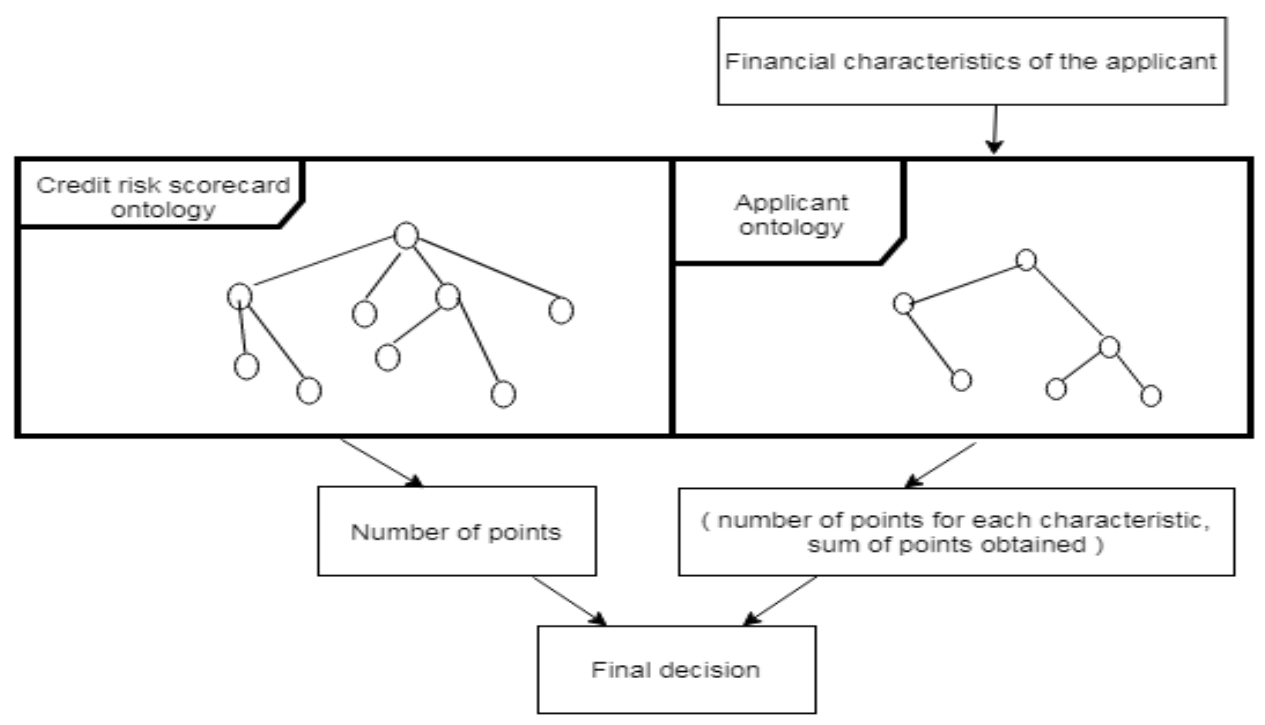

Figure 11. Reasoning algorithm

\subsection{Credit Riskscorecard Ontology}

This ontology, shown in Figure 12 is the core of the system. It contains information about the credit risk scorecard model as in Figure 10.

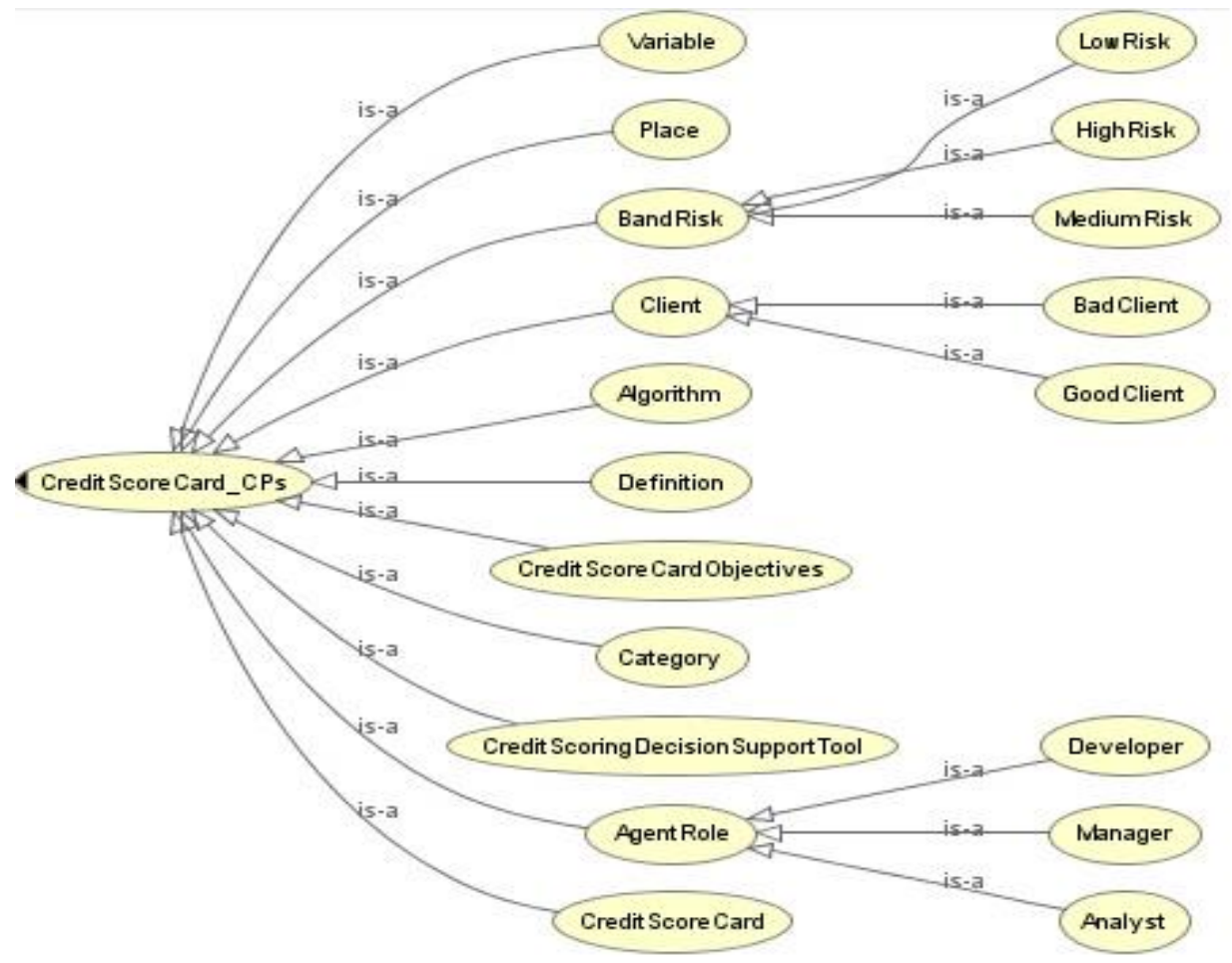

Figure 12. Credit risk scorecard ontology 


\subsection{Applicant Profile Ontology}

Applicant profile ontology, shown in Figure 13, is an OWL file that encapsulate applicant details as entered by the credit risk scorecard analyst. This file is generated as soon as the applicant submits the loan. The concept applicant profile has 20 properties: these attributes are presented in Table 2.

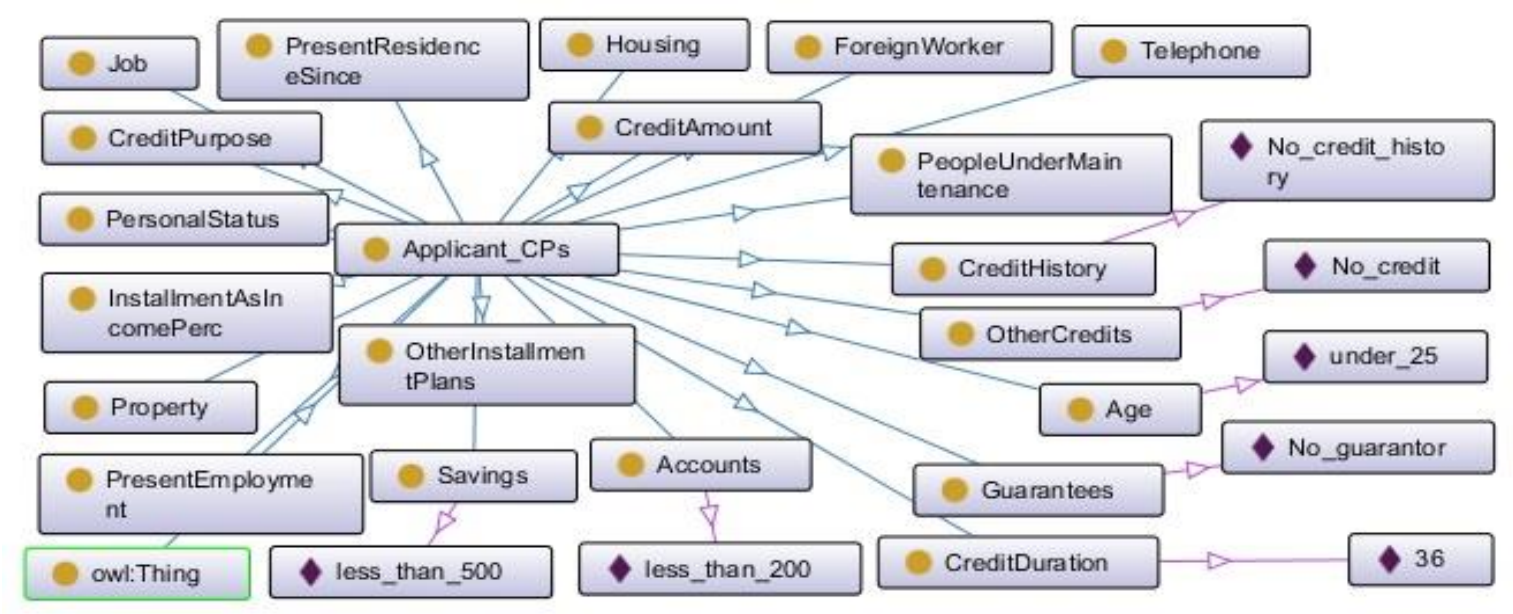

Figure 13. Applicant ontology

Table 2. The applicant profile - list of attributes

\begin{tabular}{|c|c|c|}
\hline Type of attribute & Attribute & Type \\
\hline \multirow[t]{5}{*}{ Credit information } & Accounts & Qualitative \\
\hline & Credit duration & Numerical \\
\hline & Credithistory & Qualitative \\
\hline & Creditpurpose & Qualitative \\
\hline & Creditamount & Numerical \\
\hline \multirow{3}{*}{$\begin{array}{l}\text { Applicant personnel } \\
\text { profile }\end{array}$} & Personalstatus & Qualitative \\
\hline & Age in years & Numerical \\
\hline & Telephone & Qualitative \\
\hline \multirow{12}{*}{$\begin{array}{l}\text { Applicantfinancial } \\
\text { information }\end{array}$} & Savings & Qualitative \\
\hline & Presentemployment & Qualitative \\
\hline & Installment rate in percentage of disposable income & Numerical \\
\hline & Guarantees & Qualitative \\
\hline & Presentresidencesince & Numerical \\
\hline & Property & Qualitative \\
\hline & Otherinstallment plans & Qualitative \\
\hline & Housing & Qualitative \\
\hline & Othercredits & Numerical \\
\hline & Job & Qualitative \\
\hline & Number of people being liable to provide maintenance for & Numerical \\
\hline & Foreignworker & Qualitative \\
\hline
\end{tabular}

\subsection{Ontology Testing and Result}

The first step is the analysis of the financial characteristics of the applicant. It assigns the appropriate number of points (weight) for each characteristic (Age, Other_credits, Acounts, Credit_duration, Savings, Guarantees, Credit_history) based on the credit risk scorecard ontology. By way of example, we will calculate the number of points for each characteristic for two applicants that are detailed in CQs 8 and 9 above. For instance, the applicant variable Age is divided into two categories : $>25$ years and $<=25$ years. Every characteristic has a calculated number of points. Table 3 shows the weight, and sum of points obtained for the two applicants' characteristics.

Table 3. Sample of points for applicants' characteristics

\begin{tabular}{ccccccccc}
\hline Charcteristic Applicant & Age & Other_credits & Acounts & Credit_duration & Savings & Guarantees & Credit_history & sum \\
\hline 1 (CQ 8) & 8 & 0 & 19 & 13 & 8 & 21 & 0 & 69 \\
2 (CQ 9) & 0 & 7 & 13 & 0 & 0 & 21 & 0 & 41 \\
\hline
\end{tabular}


The second step deals with decision making by comparing two parameters: the sum of points obtained and the Nbpoints in credit risk scorecard ontology. At 69 points, the first applicant reached a medium risk, while the second applicant, with 41 points, has a low risk.

The main differentiating component for this approach is the use of an ontology for representing the domain knowledge. This ontology has been used to guide the design of the solution and to supply the system with the semantic possibilities, which makes credit data meaningful. The whole approach helps the entire financial institution, from analyst to manager to understand both the business opportunities and the power of what can be gleaned from the data.

\section{CONCLUSION AND FUTURE WORK}

In this paper we used an ontology design patterns (ODPs) for modeling credit risk scorecard to improve the credit risk management. The modeling of our ontology will directly link credit risk scorecard to credit risk scorecard objectives and credit risk scoring decision support tool. The ontology is then used to identify the corresponding tasks, subtasks, roles, actors and resources of the affected business. Our proposal is expandable and aims at satisfying various needs; it provides modular, reusable, replaceable pieces, will make the data publication simpler and graph structures intuitive.In the meantime, the ontology that we developed havebeen made available on the Web as OWL file, where specific financial institutions (e.g. Credit bureau) may then use and augment it, as we are currently working on submitting it in the ontology design patterns (ODPs) portal. To enrich our proposal in the future work, we will define ontology's classes (e.g. credit risk scorecard) via axiomatization. The axiomatic method constitutes a common framework for the discussion of scientific problems for people coming from different backgrounds and even for people working in different but strongly related branches of the same discipline (e.g. credit risk scorecard analyst, manager and developer).In the end, we suggest that the proposal presented in this paper is a stepping stone towards a more complex and sophisticated credit risk ontology. For instance, extensions may consist of the development of credit risk scorecardontology as shown in Figure 4 and recommendation of credit product ontology.

\section{REFERENCES}

[1] B. DuCharme, "Learning SPARQL: Querying and Updating with SPARQL 1.1," California: Sebastopol, O’Reilly Media, 2011.

[2] A. De Nicolaa, M. Missikoff and R. Naviglib, "A software engineering approach to ontology building," Information Systems, vol. 34, p. 258-275, 2009.

[3] E. Blomqvist and K. Sandkuhl, "Patterns in ontology engineering: classification of ontology patterns," in 7th International Conference on Enterprise Information Systems, Miami, 2005.

[4] A. Gangemi, "Ontology Design Patterns for Semantic Web Content," in The Semantic Web-ISWC, Galway, 2005.

[5] E. Blomqvist, P. Hitzler, K. Janowicz, A. Krisnadhi, T. Narock and M. Solanki, "Considerations regarding ontology design patterns," Semantic Web, p. 1-7, 2016.

[6] A. Gangemi and V. Presutti, "Ontology Design Patterns," in Handbook on Ontologies, 2nd edn, 2009.

[7] K. Hammar, "Ontology design patterns in webprotege," in 14th International Semantic Web, 2015.

[8] V. Presutti, E. Daga, A. Gangemi and E. Blomqvist, "eXtreme Design with Content Ontology Design Patterns," Washington D.C, 2009.

[9] A. Krisnadhi and P. Hitzler, "Modeling with ontology design patterns: chess games as a worked example," in Ontology Engineering with Ontology Design Patterns: Foundations and Applications, vol. 25, 2016, pp. 3 - 21.

[10] P. Hitzler, K. Janowicz and K. Adila, "Ontology Modeling with Domain Experts: The GeoVocamp Experience," Diversity++@ISWC,pp. 31-36, 2015.

[11] K. Hammar, "Ontology design patterns: Adoption challenges and solutions," in WaSABi 2014, 2014.

[12] M. Alruqimi and N. Aknin, "Enabling social web for IoT inducing ontologies," International Journal of Informatics and Communication Technology (IJ-ICT), vol. 8, no. 1, pp. 19-24, April 2019.

[13] S. B. Kotsiantis, D. Kanellopoulos, V. Karioti and V. Tampakas, "An ontology-based portal for credit risk analysis," in 2009 2nd IEEE International Conference on Computer Science and Information Technology, Beijing, 2009.

[14] H. Ahuja and R. Dr. Sivakumar, "Implementation of FOAF, AIISO and DOAP ontologies for creating an academic community network using semantic frameworks," International Journal of Electrical and Computer Engineering (IJECE), vol. 9, no. 5, pp. 4302-4310, October 2019.

[15] R. Gunawan and K. Mustofa, "Finding Knowledge from Indonesian Traditional Medicine using Semantic Web Rule Language," International Journal of Electrical and Computer Engineering (IJECE), vol. 7, no. 6, pp. 3674-3682, December 2017. 
[16] F. Sidi, I. Ishak and M. A. Jabar, "MalayIK: An Ontological Approach to Knowledge Transformation in Malay Unstructured Documents," International Journal of Electrical and Computer Engineering (IJECE), vol. 8, no. 1, pp. 1-10, February 2018.

[17] J. Elhassouni, M. Bazzi, A. El Qadi and M. El Haziti, "Applying ontologies to data integration systems for bank credit risk management," Journal of Data Mining and Digital Humanities, 2018.

[18] S. Arsovski, B. Markoski, P. Pecev, L. Ratgeber and N. Petrov, "An ontology driven credit risk scoring model," in IEEE 15th International Symposium on Computational Intelligence and Informatics (CINTI), 2014.

[19] F. Polizel, S. J. Casare and J. S. Sichma, "OntoBacen: A Modular Ontology for Risk Management in the Brazilian Financial System," in JOWO@ IJCAI 2015, 2015.

[20] P. C. Sherimon and R. Krishnan, "OntoDiabetic: An Ontology -Based Clinical Decision Support System for Diabetic Patients," Arabian Journal for Science and Engineering, vol. 41 Issue 3, pp. 1145-1160, 2016.

[21] G. Mansingh and L. Rao, "Enhancing the Decision Making Process: An Ontology-based Approach," in the International Conference on Information Resources Management (Conf-IRM), 2014

[22] J. A. Morente-Molinera, R. Wikström, E. Herrera-Viedma and C. Carlsson, "A linguistic mobile decision support system based on fuzzy ontology to facilitate knowledge mobilization," Decision Support Systems, vol. 81, pp. 6675, 2016.

[23] T. P. Sales, F. A. Baião, G. Guizzardi, J. P. A. Almeida, N. Guarino and J. Mylopoulos, "The common ontology of value and risk," in 37th Int. Conf. on Conceptual Modeling (ER 2018), 2018.

[24] R. J. Feldman, "Small Business Loans, Small Banks and a Big Change in Technology Called Credit Scoring," The Region, pp. 19- 25, 1997.

[25] N. Siddiqi, Intelligent Credit Scoring: Building and Implementing Better Credit Risk Scorecards, Wiley, 2017.

[26] S. Tufféry, Data Mining and Statistics for Decision Making Methods, J. W. a. Sons, Ed., 2011.

[27] A. Krisnadhi, Y. Hu, K. Janowicz, P. Hitzler, R. Arko, S. Carbotte, C. Chandler, M. Cheatham, D. Fils, T. Finin, P. Ji, M. Jones, N. Karima, K. Lehnert, A. Mickle, T. Narock, M. O’Brien, L. Raymond, A. Shep, M. Schildhauer and P. Wiebe, "The GeoLink Modular Oceanography Ontology," in The Semantic Web - ISWC 2015, 2015.

[28] J. Raad and C. Cruz, "A Survey on Ontology Evaluation Methods," in International Conference on Knowledge Engineering and Ontology Development, part of the 7th International Joint Conference on Knowledge Discovery, Knowledge Engineering and Knowledge Management, Lisbon, Portugal, 2015.

[29] M. P. Villalón, "Ontology evaluation: a pitfall-based approach to ontology diagnosis," 2016.

[30] B. Glimm, I. Horrocks, B. Motik, G. Stoilos and Z. Wang, "HermiT: an OWL 2 reasoner," Automat. Reason, p. 1$25,2014$.

[31] H. Knublauch, R. W. Fergerson, N. F. Noy and M. A. Musen, "The Protégé OWL plugin: An open development environment for semantic web applications," in 3rd International Semantic Web Conference (ISWC), Hiroshima, Japan, 2004. 